J Am Chem Soc. 2016 July 13; 138(27): 8538-8546. doi:10.1021/jacs.6b03983.

\title{
Structure of the dimerization interface in the mature HIV-1 capsid protein lattice from solid state NMR of tubular assemblies
}

\author{
Marvin J. Bayro and Robert Tycko* \\ Laboratory of Chemical Physics, National Institute of Diabetes and Digestive and Kidney \\ Diseases, National Institutes of Health, Bethesda, MD 20892-0520
}

\section{Abstract}

The HIV-1 capsid protein (CA) forms the capsid shell that encloses RNA within a mature HIV-1 virion. Previous studies by electron microscopy have shown that the capsid shell is primarily a triangular lattice of CA hexamers, with variable curvature that destroys the ideal symmetry of a planar lattice. The mature $\mathrm{CA}$ lattice depends on $\mathrm{CA}$ dimerization, which occurs through interactions between helix 9 segments of the C-terminal domain (CTD) of CA. Several highresolution structures of the CTD-CTD dimerization interface have been reported, based on x-ray crystallography and multidimensional solution nuclear magnetic resonance (NMR), with significant differences in amino acid sidechain conformations and helix 9-helix 9 orientations. In a structural model for tubular CA assemblies based on cryogenic electron microscopy (cryoEM) [Zhao et al. (2013) Nature 497:643-646], the dimerization interface is substantially disordered. The dimerization interface structure in noncrystalline CA assemblies and the extent to which this interface is structurally ordered within a curved lattice have therefore been unclear. Here we describe solid state NMR measurements on the dimerization interface in tubular CA assemblies, which contain the curved triangular lattice of a mature virion, including quantitative measurements of intermolecular and intramolecular distances using dipolar recoupling techniques, solid state NMR chemical shifts, and long-range sidechain-sidechain contacts. When combined with restraints on the distance and orientation between helix 9 segments from the cryoEM study, the solid state NMR data lead to a unique high-resolution structure for the dimerization interface in the noncrystalline lattice of CA tubes. These results demonstrate that CA lattice curvature is not dependent on disorder or variability in the dimerization interface. This work also demonstrates the feasibility of local structure determination within large noncrystalline assemblies formed by highmolecular-weight proteins, using modern solid state NMR methods.

\section{Graphical Abstract}

\footnotetext{
*Corresponding author: robertty@mail.nih.gov.

Supporting Information

The Supporting Information is available free of charge on the ACS Publications website at DOI: 10.1021/jacs.6b03983.

- $\quad$ Summary of solid state NMR measurement conditions (Table S1)

- $\quad$ Summary of structural restraints in Xplor-NIH calculations (Table S2)

- $\quad$ Solid state NMR pulse sequences (Figure S4)

- $\quad$ Additional solid state NMR spectra (Figures S1, S2, S3, S5, and S6)

- $\quad$ Additional structure calculation results (Figures S7 and S8)
} 


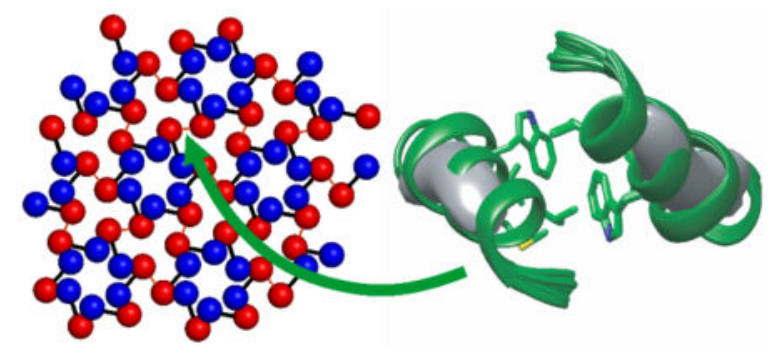

\section{INTRODUCTION}

The mature state of the type 1 human immunodeficiency virus (HIV-1) is characterized by a capsid core that encloses the viral RNA and readies the virion for infection of a new host cell. ${ }^{1}$ The capsid shell is composed of about 1500 copies of the 231 -residue capsid protein $(\mathrm{CA})^{2}$, which contains independently folding N-terminal and C-terminal domains (NTD and CTD), separated by a short linker. In solution, CA dimerizes via interactions between CTD subunits. ${ }^{3}$ In supramolecular assemblies, CA forms hexamers that are stabilized by intermolecular NTD-NTD and NTD-CTD interactions and linked to one another by CTDCTD dimerization. ${ }^{4}$

Much of our understanding of the supramolecular architecture of the mature capsid shell comes from studies of CA assemblies that form in vitro. $2,3 \mathrm{c}, 4 \mathrm{a}, 4 \mathrm{~b}, 4 \mathrm{~d}, 5$ Tubular CA assemblies (Fig. 1A) are particularly valuable models for mature capsids, as they exhibit the variable surface curvature and imperfect symmetry of mature capsids. CA tubes contain the characteristic triangular lattice of CA hexamers $3 \mathrm{c}, 4 \mathrm{a}, 4 \mathrm{~d}$ (Fig. 1B). Studies of native virions by cryogenic electron microscopy (cryoEM) confirm that the same lattice exists in the mature capsid core ${ }^{6}$, although with the likely addition of CA pentamers to permit closure of the lattice. $^{4 \mathrm{~d}, 7}$

High-resolution structural models from solution nuclear magnetic resonance (NMR) ${ }^{3 \mathrm{c}, 8}$ and $\mathrm{x}$-ray crystallography $3 \mathrm{a}, 3 \mathrm{~b}, 4 \mathrm{c}, 4 \mathrm{e}, 7 \mathrm{~b}, 9$ have brought a molecular understanding to the extensive biochemical and mutagenic studies on the function of CA and its mechanism of assembly. $2,3 \mathrm{c}, 10$ The dimerization interface, formed by hydrophobic residues in helix 9 segments of CTD (residues 179-193 of full-length CA), is especially critical. Amino acid substitutions in helix 9 result in non-infectious virions in vivo ${ }^{10}$, due to disruption of the HIV-1 maturation process, and preclude CA lattice assembly and tube formation in vitro. $2,8 \mathrm{c}, 8 \mathrm{~d}, 11$

According to earlier studies, intermolecular interactions involving W184 and M185 are the main contributors to the stability of the dimerization interface. ${ }^{3,4 e}$ A variety of conformations have been observed in the various constructs utilized in these studies. As shown in Fig. 1C, the orientation of W184 sidechains in a crystal structure of CTD (residues 146-231, PDB 1A43 ${ }^{3 \mathrm{~b}}$ is clearly different from the orientation in a solution NMR structure of CTD (PDB 2KOD). ${ }^{3 \mathrm{c}}$ Although the dimerization interface in a recent crystal structure of full-length CA (PDB 4XFX) ${ }^{4 e}$ is similar to that in the solution NMR structure of CTD, sidechain conformations and helix 9-helix 9 orientations are somewhat different (e.g., intra- 
residue M185 $\mathrm{C}_{\mathrm{a}}-\mathrm{C}_{\varepsilon}$ and intermolecular $\mathrm{L} 189 \mathrm{C}_{\mathrm{a}}-\mathrm{L} 189 \mathrm{C}_{\mathrm{a}}$ distances of $5.3 \AA$ and $11.4 \AA$ in PDB 4XFX, compared to 4.4-5.2 $\AA$ and 14.2 $\AA$ in PDB 2KOD). Moreover, the relevance of structural results from solution NMR and crystallography to noncrystalline assemblies of full-length CA has been unclear. In a recent model for CA tubes developed from cryoEM (EMDB-5582 and PDB 3J34) ${ }^{4 d}$, sidechain conformations and helix 9-helix 9 distances in the dimerization interface are significantly different and highly disordered, with large variations from one CTD-CTD interface to another (see below). These differences from structural studies in different contexts suggest that the dimerization interface may have inherent structural plasticity, which may contribute to the variable curvature of the CA lattice in mature capsids. ${ }^{3 c, 4 d, 12}$ On the other hand, solid state NMR spectra of CA tubes show a single set of sharp signals for residues in the dimerization interface, indicating a unique and well-defined molecular structure even in non-planar, noncrystalline CA assemblies ${ }^{13}$

Here we describe solid state NMR experiments on HIV-1 CA tubes aimed at elucidating the structure of the dimerization interface in noncrystalline assemblies at atomic resolution. By combining a variety of residue-specific isotopic labeling patterns (Table 1) with a variety of solid state NMR techniques, we obtain a set of quantitative restraints on the dimerization interface structure. When supplemented with restraints on the distance and relative orientation between helix 9 segments from the reported cryoEM density of CA tubes ${ }^{4 \mathrm{~d}}$, the solid state NMR data are sufficient to determine a unique dimerization interface structure, which represents the first high-resolution characterization of an intermolecular interface in noncrystalline HIV-1 CA assemblies that mimic the mature HIV-1 capsid.

\section{MATERIALS AND METHODS}

\section{Sample preparation}

HIV-1 CA protein (plasmid pNL4-3) was expressed and purified as described previously. ${ }^{13}$ Purified protein solutions were concentrated to $30 \mathrm{mg} / \mathrm{ml}$ in Tris buffer ( $50 \mathrm{mM}, \mathrm{pH} 8.0$ ) prior to addition of $\mathrm{NaCl}$ to $1.0 \mathrm{M}$ concentration to promote self-assembly. The tubular morphology and sample homogeneity were verified with TEM images, using an FEI Morgagni microscope operating at $80 \mathrm{keV}$. All CA samples were grown in media containing unlabeled glucose, except samples II and III, which used $2-{ }^{13} \mathrm{C}$-glycerol as the carbon source. Both samples mixed to make sample IV were produced using unlabeled $\mathrm{NH}_{4} \mathrm{Cl}$ as the nitrogen source. For amino-acid-specific labeling, the appropriate precursors were added to the cell culture one hour before induction, including $\mathrm{U}-{ }^{13} \mathrm{C},{ }^{15} \mathrm{~N}-\mathrm{L}$-methionine, ${ }^{13} \mathrm{C}_{\varepsilon}$ - $\mathrm{L}$ methionine, $2-{ }^{13} \mathrm{C}$-indole (for labeling of $\operatorname{Trp} \mathrm{C}_{\delta 1}$ ), or ${ }^{15} \mathrm{~N}$-indole $(120 \mathrm{mg} / \mathrm{l}$ each). For sample III, aromatic ${ }^{13} \mathrm{C}$ signals from Tyr and Phe residues were suppressed by supplying unlabeled L-tyrosine and L-phenylalanine ( $150 \mathrm{mg} / \mathrm{l}$ of each amino acid). Absence of Tyr and Phe signals from solid state NMR spectra of sample III confirmed their reverse labeling.

For sample V, mass spectrometry indicates $98 \%$ incorporation of ${ }^{15} \mathrm{~N}$ at amide sites of the 11 Met residues, through aminotransferase-catalyzed exchange with ${ }^{15} \mathrm{~N}$-labeled amino acids. Specifically, the molecular mass of sample V was determined experimentally to be 25924 $\mathrm{Da}$, compared with an experimental value of $25913 \mathrm{Da}$ for CA with only ${ }^{15} \mathrm{~N}$ labeling. Uncertainties in molecular masses were $0.2 \mathrm{Da} .{ }^{13} \mathrm{C}$ labeling patterns for samples II and III were deduced from $2 \mathrm{D}{ }^{13} \mathrm{C}-{ }^{13} \mathrm{C}$ spectra, and were found to be in general agreement with 
expectations based on earlier publications. ${ }^{14}$ However, in addition to the expected $\mathrm{C}_{\gamma}, \mathrm{C}_{\delta 2}$, and $C_{\zeta 3}$ sites of Trp residues, signals from $C_{\delta 1}$ and $C_{\varepsilon 3}$ were also observed.

\section{Solid state NMR spectroscopy}

2D NMR spectra, rotational echo double resonance ${ }^{15}$ (REDOR) data, and $\mathrm{NCCN}^{16}$ data were obtained with a Varian InfinityPlus spectrometer operating at a ${ }^{1} \mathrm{H}$ NMR frequency of 599.2 MHz, equipped with a Varian BioMAS probe with a 3.2-mm MAS module. LeeGoldburg cross-polarization ${ }^{17}$ (LG-CP) and broadband rotational resonance ${ }^{18}$ (BroBaRR) data were obtained with a Varian Infinity spectrometer operating at a ${ }^{1} \mathrm{H}$ NMR frequency of 746.4 MHz, equipped with a Bruker Efree probe with a 3.2-mm MAS module. Sample temperatures were maintained near $20^{\circ} \mathrm{C}$ with cooled nitrogen gas. $2 \mathrm{D}{ }^{13} \mathrm{C}-{ }^{13} \mathrm{C}$ and ${ }^{15} \mathrm{~N}-{ }^{13} \mathrm{C}-{ }^{13} \mathrm{C}$ correlation spectra were recorded with magic-angel spinning (MAS) at $11.00 \mathrm{kHz}$ (sample I) or $16.00 \mathrm{kHz}$ (samples II and III), using spin diffusion mixing periods $\tau_{\mathrm{SD}}=200 \mathrm{~ms}$ (sample I) or $\tau_{\mathrm{SD}}=700 \mathrm{~ms}$ (samples II and III) for ${ }^{13} \mathrm{C}-{ }^{13} \mathrm{C}$ longitudinal exchange, 3.0 ms periods for ${ }^{15} \mathrm{~N}-{ }^{13} \mathrm{C}$ polarization transfer by cross-polarization, and 80 $\mathrm{kHz}$ proton decoupling levels, with two-pulse phase modulation(ref) (TPPM). ${ }^{1} \mathrm{H}_{-}{ }^{13} \mathrm{C}$ LG$\mathrm{CP}$ measurements used $11.00 \mathrm{kHz}$ MAS, a $50 \mathrm{kHz}{ }^{13} \mathrm{C}$ rf field amplitude, and a $61 \mathrm{kHz}$ effective ${ }^{1} \mathrm{H}$ field. ${ }^{1} \mathrm{H}^{-15} \mathrm{~N}$ LG-CP measurements used $11.00 \mathrm{kHz}$ MAS, a $30 \mathrm{kHz}{ }^{15} \mathrm{~N}$ rf field amplitude, and a $41 \mathrm{kHz}$ effective ${ }^{1} \mathrm{H}$ field. REDOR data were recorded with $8.93 \mathrm{kHz}$ MAS, using $85 \mathrm{kHz}$ proton decoupling with TPPM and $12.5 \mu \mathrm{s}{ }^{15} \mathrm{~N} \pi$ pulses during the REDOR recoupling period. BroBaRR experiments were performed with $8.00 \mathrm{kHz}$, to match approximately the chemical shift difference between M185 $C_{a}$ and $C_{\varepsilon}$ resonances. During the BroBaRR recoupling period, the ${ }^{13} \mathrm{C}$ rf carrier frequency was set to $38.5 \mathrm{ppm}$, the effective ${ }^{13} \mathrm{C} \pi$ pulse lengths were $500 \mu$ s with MLEV-32 phases, ${ }^{19}$ and the ${ }^{1} \mathrm{H}$ decoupling level was $85 \mathrm{kHz}$. NCCN vector angle measurements were performed on sample III with REDOR recoupling conditions as described above and with $\tau_{\mathrm{SD}}=200 \mathrm{~ms}$ for ${ }^{13} \mathrm{C}-{ }^{13} \mathrm{C}$ longitudinal exchange between REDOR periods. Additional details of NMR measurements are given in Table S1.

\section{Simulations of solid state NMR data}

Numerical simulations of LG-CP data were performed with SPINEVOLUTION software. ${ }^{20}$ REDOR simulations were performed with SIMPSON. ${ }^{21} \mathrm{NCCN}$ and BroBaRR measurements were simulated with custom programs (available upon request) that calculated spin density matrix evolution under the relevant $\mathrm{rf}$ pulse sequences, with time-dependent dipole-dipole couplings under MAS. In NCCN simulations, ${ }^{13} \mathrm{C}-{ }^{13} \mathrm{C}$ polarization transfer was assumed to be orientation-independent. BroBaRR simulations included transverse $\left(\mathrm{T}_{2}\right)$ relaxation as damping of appropriate density matrix elements, using $\mathrm{T}_{2}$ values measured for ${ }^{13} \mathrm{C}_{a}$ and ${ }^{13} \mathrm{C}_{\varepsilon}$ under the BroBaRR pulse sequence (7 ms and $25 \mathrm{~ms}$, respectively).

\section{Structure calculations}

Simulated annealing calculations were carried out with $\mathrm{Xplor-} \mathrm{NIH}^{22}$, using experimental restraints from solid state NMR measurements and the cryoEM density of CA tubes in the Electron Microscopy Data Bank (EMD-5582). CryoEM densities for interacting helix 9 segments were extracted within the Chimera program (https://www.cgl.ucsf.edu/chimera/), by fitting pairs of helix 9 segments (residues 175-193) from PDB 3J34 to the density and 
then using the "Zone" function to select the density within $3.0 \AA$ of the atomic coordinates. Xplor-NIH calculations included two copies of residues 175-193, with initial atomic coordinates taken from PDB files 4XFX, 2KOC, or 1A43. Prior to simulated annealing, a preliminary rigid-body fit of the initial atomic coordinates to the cryoEM density (Fig. S7) was performed, without allowing changes in interhelical distance or orientation, using the probDistPot potential energy term and the randomizeDomainPos function of Xplor-NIH. Simulated annealing was then performed from $3500 \mathrm{~K}$ to $25 \mathrm{~K}$ in 12,500 steps, with 0.2 ps of torsion angle dynamics at each temperature step. Distance restraints from REDOR and BroBaRR measurements (Figs. 3C-3F) were represented by NOE potentials with distance ranges equal to the experimental error ranges stated in the main text. Distance restraints from long-range correlation experiments (Fig. 2C) were assigned an upper limit of $7.0 \AA$. The force constant for NOE potential terms increased geometrically from 50 to $200 \mathrm{kcal} /$ mol- $\AA^{2}$ during annealing. Vector angle restraints from NCCN data (Fig. $3 \mathrm{G}$ ) were represented by VEAN potential in Xplor-NIH and a force constant increasing from 1 to 10 $\mathrm{kcal} / \mathrm{mol}^{-\mathrm{rad}^{2}}$. The cryoEM density was applied as a restraint on backbone heavy atoms only, using the probDistPot potential ${ }^{23}$ with a scale factor of $40 \mathrm{kcal} / \mathrm{mol}-\AA^{4}$, and allowing the two copies of residues 179-193 to move independently of each other. The observation of a single set of chemical shifts for each residue in solid state NMR spectra of CA tubes implies a single protein conformation. Therefore, a noncrystallographic symmetry potential (NCS) was applied, with a scale factor of $10 \mathrm{kcal} / \mathrm{mol}-\AA^{2}$. In order to preserve the integrity of the a-helical conformation, hydrogen bond restraints between residues i and $i+4$ were included in the NOE potential, for $\mathrm{i}=178-189$. In addition, backbone $\phi$ and $\psi$ torsion angle restraints from our previous work ${ }^{13}$, which are consistent with an a-helical conformation, were applied with the CDIH potential, using a scale factor of $400 \mathrm{kcal} / \mathrm{mol}_{-} \mathrm{rad}^{2}$. The scale factor for van der Waals-like repulsions increased from 0.004 to $4 \mathrm{kcal} / \mathrm{mol}-\AA^{4}$, with a radius scale factor decreasing from 0.9 to 0.8 . Standard bond length, bond angle, and improper angle potentials were also used. After annealing, energy minimization was performed. In each set of structure calculations, the 20 lowest-energy structures out of 200 independent calculations were selected for each structure ensemble.

\section{RESULTS}

\section{Assignment of solid state NMR signals in the dimerization interface}

In a previous study ${ }^{13}$, we obtained ${ }^{15} \mathrm{~N}$ and ${ }^{13} \mathrm{C}$ chemical shift assignments for most backbone $\mathrm{N}, \mathrm{CO}, \mathrm{C}_{\mathrm{a}}$, and $\mathrm{C}_{\beta}$ sites in $\mathrm{CA}$ tubes, as well as some sidechain assignments. Additional sidechain assignments, especially for $\mathrm{W} 184$ and M185, were required for the structural measurements described below. To obtain ${ }^{13} \mathrm{C}$ chemical shift assignments for Met sidechains, we prepared a CA tube sample that was uniformly ${ }^{15} \mathrm{~N}$-labeled at all residues and uniformly ${ }^{13} \mathrm{C}$-labeled only at Met residues (sample I). From 2D NCACX and NCOCX spectra (Fig. S1) and $2 \mathrm{D}^{13} \mathrm{C}-{ }^{13} \mathrm{C}$ spectra (Fig. 2A, Fig. S2), we obtained full assignments for eight of the 11 Met residues in CA, including M185. Importantly, the ${ }^{13} \mathrm{C}_{\varepsilon}$ signal of M185 is resolved from those of all other Met residues.

Chemical shift assignments for aromatic sidechains were obtained from $2 \mathrm{D}{ }^{13} \mathrm{C}-{ }^{13} \mathrm{C}$ spectra of CA tubes that were partially ${ }^{13} \mathrm{C}$-labeled (sample II), using $2-{ }^{13} \mathrm{C}$-glycerol as the carbon 
source for protein expression (Fig. 2B). Most carbon sites in Trp sidechains were partially ${ }^{13} \mathrm{C}$-labeled in this sample, allowing us to trace connectivities among sidechain signals for W184, as well as for the other Trp residues. Additional spectra of a sample labeled with $2-{ }^{13} \mathrm{C}$ glycerol and reverse-labeled with natural-abundance Tyr and Phe amino acids (sample III) helped us identify partially overlapping aromatic signals.

\section{Rigidity of the dimerization interface}

Before attempting quantitative structural measurements, it was important to establish that sidechains in the dimerization interface are not highly dynamic. Large-amplitude motions of sidechains on sub-millisecond time scales attenuate one-bond ${ }^{1} \mathrm{H}_{-}^{13} \mathrm{C}$ and ${ }^{1} \mathrm{H}_{-}{ }^{15} \mathrm{~N}$ dipoledipole couplings in solid state NMR measurements on proteins. ${ }^{24}$ Therefore, we measured the one-bond ${ }^{1} \mathrm{H}_{-}{ }^{13} \mathrm{C}_{\varepsilon}$ coupling for M185 and the one-bond ${ }^{1} \mathrm{H}_{-}-{ }^{15} \mathrm{~N}_{\varepsilon 1}$ coupling for W184 in CA tubes, using the LG-CP technique. ${ }^{17}$ As shown in Figs. 3A and 3B, build-up curves for M185 ${ }^{13} \mathrm{C}_{\varepsilon}$ and $\mathrm{W} 184{ }^{15} \mathrm{~N}_{\varepsilon 1}$ signals in CA tubes under LG-CP are nearly identical to buildup curves for amino acid powders, in which large-amplitude sidechain motions are not present, and are in good agreement with numerical simulations for rigid sites (but with rapid uniaxial methyl rotation). Together with the observation of sharp crosspeak signals for M185 and W184 (Figs. 2A and 2B), the LG-CP data imply a well-defined, rigid molecular structure in the dimerization interface. In contrast, the M185 ${ }^{13} \mathrm{C}_{\varepsilon}$ signal is absent from solution NMR spectra of soluble CA dimers, presumably due to conformational exchange (Fig. S3).

In addition, one-dimensional ${ }^{13} \mathrm{C}$ and ${ }^{15} \mathrm{~N}$ solid state NMR spectra showed no significant changes in signal intensities from M185 and W184 sidechain over the $0-30^{\circ} \mathrm{C}$ temperature range. Previously reported $2 \mathrm{D}{ }^{13} \mathrm{C}-{ }^{13} \mathrm{C}$ spectra of $\mathrm{CA}$ tubes, acquired with conditions similar to those in solution NMR (i.e., low-power proton decoupling and spin polarization transfers driven by scalar couplings), did not show signals attributable to M185 or W184, indicating an absence of rapid, isotropic reorientation.

\section{Quantitative structural restraints from solid state NMR}

Quantitative restraints on specific interatomic distances in protein assemblies can be obtained from quantitative measurements of nuclear magnetic dipole-dipole couplings, using a class of solid state NMR techniques with magic-angle spinning (MAS) called "dipolar recoupling" techniques. ${ }^{25}$ These techniques require that signals from the sites of interest be sufficiently well resolved that their intensities can be measured accurately. For this purpose, we used samples I, IV, and V, which allowed us to measure specific intermolecular and intraresidue distances involving W184 and M185.

The intermolecular M185 $\mathrm{C}_{\varepsilon}-\mathrm{W} 184 \mathrm{~N}_{\varepsilon 1}$ distance across the dimerization interface was measured with REDOR. ${ }^{15 a}$ Sample IV was used for these measurements to ensure that only intermolecular ${ }^{15} \mathrm{~N}_{-}{ }^{13} \mathrm{C}$ couplings were measured and that the detected $\mathrm{M} 185{ }^{13} \mathrm{C}_{\varepsilon}$ signals were resolved in 1D. REDOR data are plotted in Fig. $3 \mathrm{C}$ as the normalized difference $\left(\mathrm{S}_{0^{-}}\right.$ $\left.\mathrm{S}_{1}\right) / \mathrm{S}_{0}$, where $\mathrm{S}_{1}$ and $\mathrm{S}_{0}$ are ${ }^{13} \mathrm{C}$ NMR signal areas with and without a central ${ }^{15} \mathrm{~N} \pi$ pulse that determines whether a net recoupling of ${ }^{15} \mathrm{~N}-{ }^{13} \mathrm{C}$ interactions occurs (Fig. S4A). The data are additionally scaled by a factor of 2.0 to account for the $50 \%{ }^{15} \mathrm{~N}$ labeling in sample 
IV. As shown in Fig. 3C, the REDOR data can be fit with simulations for a ${ }^{15} \mathrm{~N}-{ }^{13} \mathrm{C}$ distance of 3.5-4.0 $\AA$. This distance agrees well with the $4.1 \AA$ intermolecular $\mathrm{M} 185 \mathrm{C}_{\varepsilon}-\mathrm{W} 184 \mathrm{~N}_{\varepsilon 1}$ distance in PDB 4XFX (2.43 $\AA$ crystallographic resolution), but is shorter than distances in PDB 2KOD (NMR structure with $0.9 \AA$ heavy atom root-mean-squared deviation) (4.3-5.2 $\AA$ ) and PDB 1A43 (2.60 ̊̊ crystallographic resolution) $(7.5 \AA)$.

Intra-residue $\mathrm{M} 185 \mathrm{C}_{\varepsilon}-\mathrm{N}$ and $\mathrm{W} 184 \mathrm{C}_{\delta 1}-\mathrm{N}$ distances, which restrain the $\mathrm{M} 185$ and $\mathrm{W} 184$ sidechain conformations, were also measured with REDOR. Sample V, in which only Met $\mathrm{C}_{\varepsilon}$ and $\operatorname{Trp} \mathrm{C}_{\delta 1}$ sites were ${ }^{13} \mathrm{C}$-labeled and all backbone nitrogens (but not Trp sidechain nitrogens) were ${ }^{15} \mathrm{~N}$-labeled, was used. Experimental $\left(\mathrm{S}_{0}-\mathrm{S}_{1}\right) / \mathrm{S}_{0}$ data are shown in Figs. 3D and $3 \mathrm{E}$, without additional scaling. In these measurements, depending on the sidechain conformations, inter-residue ${ }^{15} \mathrm{~N}-{ }^{13} \mathrm{C}$ couplings involving backbone nitrogens of neighboring residues are not necessarily negligible. Therefore, three-spin simulations were performed, including the intra-residue ${ }^{15} \mathrm{~N}-{ }^{13} \mathrm{C}$ pair and one additional ${ }^{15} \mathrm{~N}$ spin at a variable position chosen to be consistent with a-helical structure in residues 183-186. Solid and dashed lines in Figs. 3D and 3E represent simulations with the additional ${ }^{15} \mathrm{~N}$ spin at positions that produce the most and least rapid build-up of REDOR difference signals, respectively. Comparisons of simulations with experimental data then indicated an intraresidue $\mathrm{M} 185 \mathrm{C}_{\varepsilon}-\mathrm{N}$ distance of 4.3-5.0 $\AA$ and an intra-residue $\mathrm{W} 184 \mathrm{C}_{\varepsilon 1}$ - $\mathrm{N}$ distance of 4.0$5.0 \AA$ A. For comparison, M185 $\mathrm{C}_{\varepsilon}-\mathrm{N}$ distances in PDB 4XFX, 2KOD, and $1 \mathrm{~A} 43$ are $5.7 \AA$, 4.8-5.7 $\AA$, and 5.1 $\AA$, respectively. W184 $\mathrm{C}_{\delta 1}-\mathrm{N}$ distances in PDB 4XFX, 2KOD, and $1 \mathrm{~A} 43$ are $4.7 \AA$. $4.5-4.6 \AA$, and $3.6 \AA$.

Experimental REDOR data in Figs. 3D and 3D clearly deviate from the simulated curves at larger values of the evolution time. This effect is attributable to insufficiently large proton decoupling fields ${ }^{26}$, which lead to an accumulating imperfection in ${ }^{15} \mathrm{~N}$ spin inversion by the train of MAS-synchronized ${ }^{15} \mathrm{~N} \pi$ pulses in the REDOR sequence (Fig. S4A). The uncertainties in ${ }^{15} \mathrm{~N}-{ }^{13} \mathrm{C}$ distances stated above take this effect into account.

The intra-residue M185 $\mathrm{C}_{\mathrm{a}}-\mathrm{C}_{\varepsilon}$ distance was measured with BroBaRR ${ }^{18}$, which allows the dipole-dipole coupling between two specific ${ }^{13} \mathrm{C}$-labeled sites to be measured in a uniformly ${ }^{13} \mathrm{C}$-labeled residue, provided that at least one of the two ${ }^{13} \mathrm{C}$ chemical shifts is resolved. Selectivity in the BroBaRR technique is achieved by proper choice of the ${ }^{13} \mathrm{C}$ radio-frequency (rf) carrier frequency, the MAS frequency, and the amplitude of a train of weak rf pulses that is applied during the BroBaRR evolution period (Fig. S4B).

Experimental BroBaRR data, obtained with sample I, are compared with simulations in Fig. 3F. For M185, the $\mathrm{C}_{\mathrm{a}}-\mathrm{C}_{\varepsilon}$ distance was found to be 3.5-4.3 $\mathrm{A}$, shorter than the $5.3 \AA$ and 4.4-5.2 $\AA$ distances in PDB 4XFX and 2KOD, and similar to the $4.1 \AA$ distance in PDB 1A43. BroBaRR data for M68 were obtained simultaneously, indicating a $\mathrm{C}_{\mathrm{a}}-\mathrm{C}_{\varepsilon}$ distance of $4.9+/-0.2 \AA$ that is in good agreement with the corresponding distances of $4.5 \AA, 4.8 \AA$, and $4.9 \AA$ in crystal structures of full-length CA (PDB 4XFX, 3MGE, and 3P05). ${ }^{4 c, 4 e, 7 b}$ Representative spectra from REDOR and BroBaRR experiments are shown in Fig. S5. In addition to distance measurements with dipolar recoupling techniques, structural restraints can be obtained with "tensor correlation" techniques in solid state NMR, which measure the orientations of two different chemical bond vectors or functional groups relative to one another. ${ }^{27}$ As a further restraint on the W184 sidechain conformation in CA tubes, we 
recorded NCCN tensor correlation data ${ }^{16}$ to probe the angle between the backbone $\mathrm{C}_{\mathrm{a}}-\mathrm{N}$ bond vector and the sidechain $\mathrm{C}_{\delta 1}-\mathrm{N}_{\varepsilon 1}$ bond vector. Sample III, which was uniformly ${ }^{15} \mathrm{~N}$ labeled and partially ${ }^{13} \mathrm{C}$-labeled, but not ${ }^{13} \mathrm{C}$-labeled at Tyr and Phe residues, was chosen for these measurements because the ${ }^{13} \mathrm{C}_{\delta 1}$ signal of W184 was fully resolved. In our version of the NCCN pulse sequence (Fig. S4C), ${ }^{13} \mathrm{C}$ polarization on $\mathrm{C}_{a}$ was prepared by ${ }^{1} \mathrm{H}_{-}{ }^{15} \mathrm{~N}$ cross-polarization followed by band-selective ${ }^{15} \mathrm{~N}-{ }^{13} \mathrm{C}_{a}$ cross-polarization. After a REDOR period for evolution under the ${ }^{15} \mathrm{~N}-{ }^{13} \mathrm{C}_{a}$ dipole-dipole coupling, the polarization was transferred to $\mathrm{C}_{\delta 1}$ during a $200 \mathrm{~ms}{ }^{13} \mathrm{C}_{-13}{ }^{13} \mathrm{C}$ spin diffusion period (Fig. S6). After a second REDOR period for evolution under the ${ }^{15} \mathrm{~N}_{\varepsilon 1^{-}}{ }^{13} \mathrm{C}_{\delta 1}$ dipole-dipole coupling, ${ }^{13} \mathrm{C}_{\delta 1}$ signals were measured. Experimental NCCN data are compared with simulations in Fig. 3F. For $\mathrm{W} 184$, the data indicate an angle in the $150^{\circ}-170^{\circ}$ range (absolute value). Values of the same angle are $160^{\circ}, 164-174^{\circ}$, and $113^{\circ}$ in PDB 4XFX, 2KOD, and $1 \mathrm{~A} 43$, respectively. NCCN data were also obtained for W117, implying an angle between $\mathrm{C}_{a}-\mathrm{N}$ and $\mathrm{C}_{\delta 1^{-}}-\mathrm{N}_{\varepsilon 1}$ bond vectors that is less than $150^{\circ}$ (absolute value), in a range where the NCCN signals decay rapidly and are not sensitive to the precise angle. For comparison, values of the same angle for $\mathrm{W} 117$ in PDB 4XFX, 3MGE, and 3P05 are $78^{\circ}, 80^{\circ}$, and $77^{\circ}$.

Fig. $3 \mathrm{H}$ summarizes the aspects of the dimerization interface that are restrained by the quantitative structural measurements in Figs. 3C-3G. As discussed above, these measurements for CA tubes are not fully consistent with previous high-resolution structures, showing that the dimerization interface structure in noncrystalline CA tubes is not the same as in the soluble or crystalline constructs examined previously. The good agreement between solid state NMR data for M68 and W117 (Figs. 3F and 3G) and results from crystallography is reassuring, since both M68 and W117 are in NTD and are not involved in dimerization or other potentially variable intermolecular interfaces.

\section{Sidechain-sidechain contacts}

While W184 and M185 constitute the core of the dimerization interface, other hydrophobic residues of helix 9 segments also participate in intermolecular interactions in previously described structures. ${ }^{3,4 \mathrm{e}}$ Since various interactions are possible, depending on the sidechain conformations, we recorded additional $2 \mathrm{D}^{13} \mathrm{C}-{ }^{13} \mathrm{C}$ spectra with long spin diffusion mixing periods $\left(\tau_{\mathrm{SD}}=700 \mathrm{~ms}\right)$ to reveal sites close in space to W184. Fig. $2 \mathrm{C}$ shows the aromaticaliphatic section of a 2D spectrum of sample III, under conditions where crosspeaks are expected between ${ }^{13} \mathrm{C}$ pairs with internuclear distances up to roughly $7 \AA$. Multiple crosspeaks are observed between aromatic ${ }^{13} \mathrm{C}$ sites of W184 and aliphatic sidechain sites of V181 and L189, with signal-to-noise values in the 9-15 range. These crosspeaks help to define the conformation of W184 in the context of interactions with surrounding hydrophobic residues.

\section{Structure calculations with combined cryoEM density and solid state NMR restraints}

Structure calculations were performed by restrained simulated annealing in Xplor-NIH. ${ }^{22}$ Restraints from solid state NMR, described above, are sufficient to define the structural arrangement of hydrophobic sidechains within the dimerization interface, but do not determine the distance and orientation between the two helix 9 segments. Therefore, we made use of the cryoEM density map for CA tubes reported by Zhao et al. ${ }^{4 \mathrm{~d}}$, deposited in 
the Electron Microscopy Data Bank as file EMD-5582. Densities for helix 9 pairs (Fig. S7) were extracted manually, using the Chimera program (https://www.cgl.ucsf.edu/chimera/). These densities were applied as restraints in simulated annealing structure calculations, using the probDistPot potential energy function of Xplor-NIH. Restraints based on solid state NMR data included quantitative interatomic distance restraints from REDOR and BroBaRR data, vector angle restraints from the NCCN data (using the VEAN potential term of Xplor-NIH), upper-bound distance restraints from long-range crosspeaks in $2 \mathrm{D}^{13} \mathrm{C}-{ }^{13} \mathrm{C}$ spectra with $\tau_{\mathrm{SD}}=700 \mathrm{~ms}$, and backbone torsion angle restraints obtained previously from ${ }^{15} \mathrm{~N}$ and ${ }^{13} \mathrm{C}$ chemical shifts. ${ }^{13}$ Starting structures were pairs of interacting helix 9 segments (residues 175-193) from PDB files 4XFX, 2KOD, and 1A43 (Figs. 1C and S7AD). Thus, these calculations can be considered to be structure refinements, rather than $d e$ novo structure determinations, in which we have used structures from soluble or crystalline samples as the starting point for high-resolution characterization of the dimerization interface structure in the noncrystalline, curved lattice of CA tubes.

Fig. 4A shows a superposition of the three starting structures, highlighting the differences in helix orientations and sidechain conformations. Fig. 4B shows a superposition of the corresponding lowest-energy final structures. Pairwise root-mean-squared deviations (rmsd) among these structures, including backbone heavy atoms of residues 179-193 and sidechain heavy atoms of V181, W184, M185, and L189, are reduced from 1.35-3.50 ̊ to 0.85$1.16 \AA$. The convergence of refined structures from three different starting points demonstrates that the combination of solid state NMR and cryoEM-based restraints results in a unique result, which represents the dimerization interface structure in CA tubes.

Rmsd values (as defined above) between starting structures and lowest-energy final structures (Figs. 4A and 4B) are $0.92 \AA, 1.40 \AA$, and $3.48 \AA$ for starting structures taken from PDB 4XFX, 2KOD, and 1A43, respectively. In all cases, restrained simulated annealing results in structural changes, indicating that the dimerization interface structure in CA tubes is not identical to structures in soluble or crystalline states. In calculations starting with PDB 4XFX, the most significant changes are in the values of sidechain torsion angles for $\mathrm{W} 184$ (from $\chi_{1}=176^{\circ}, \chi_{2}=85^{\circ}$ to $\chi_{1}=165^{\circ}, \chi_{2}=92^{\circ}$ ), which result in displacements of sidechain indole atoms by as much as $1.1 \AA$. Similar displacements of other hydrophobic sidechains also occur in the dimerization interface. In calculations starting with PDB 2KOD, displacements of indole atoms are somewhat larger (up to $1.6 \AA$ ), reducing the nearestneighbor carbon-carbon distance between W184 sidechains by $1.7 \AA$. The intermolecular angle between $a$-helices also increases by approximately $8^{\circ}$. In calculations starting with PDB 1A43, sidechain conformations in the dimerization interface change dramatically, with displacements of sidechain indole atoms up to $7.0 \AA$ A. The intermolecular angle between $a$ helices increases by approximately $28^{\circ}$.

Fig. 4C shows a bundle of 20 final structures from a set of 100 independent calculations, all starting with coordinates from PDB 4XFX (see also Fig. S8E). The rmsd for all heavy atom coordinates in this bundle is $1.04 \AA$. The largest rmsd contributions come from sidechains that are not involved in intermolecular contacts, which were unrestrained. For backbone heavy atoms of all residues and sidechain heavy atoms of V181, W184, M185, and L189, the rmsd is $0.41 \AA$. Thus, the experimental data are sufficient to define the dimerization 
interface structure with high precision. Atomic coordinates from Fig. 4C have been deposited in the Protein Data Bank as PDB file 5IRT. Structural restraints and corresponding values in PDB 5IRT are summarized in Table S2.

Finally, Fig. 4D shows a superposition of six lowest-energy final structures from calculations in which six different cryoEM densities were used (Fig. S7), with the same starting structure (from PDB 4XFX) in each case. The rmsd value among these six final structures, including backbone heavy atoms of all residues and sidechain heavy atoms of V181, W184, M185, and L189, is $0.50 \AA$. Thus, the specific choice of helix 9 pair from the cryoEM density map is unimportant. This observation is consistent with our finding that the lowering of symmetry induced by lattice curvature and by the variations in CA tube diameters in solid state NMR samples does not produce significant inhomogeneous broadening of solid state NMR signals from the dimerization interface, relative to signals from other regions of CA. ${ }^{13}$ In contrast, dimerization interface structures from molecular dynamics flexible fitting (MDFF) calculations (PDB 3J34) ${ }^{4 \mathrm{~d}}$ exhibit substantially greater variability and disorder (Fig. S7), apparently reflecting the lack of restraints with sufficiently high resolution in the MDFF calculations.

\section{DISCUSSION}

Results described above demonstrate a unique role for solid state NMR in applications to noncrystalline supramolecular assemblies formed by proteins of relatively high molecular weight. For assemblies such as HIV-1 CA tubes, full structure determination based entirely on solid state NMR data would be extremely challenging, primarily due to limited resolution in the multidimensional spectra from which the necessary long-range structural restraints would be obtained. However, full structure determination is not required, since a wealth of structural information already exists from solution NMR studies of soluble NTD, CTD, and full-length $\mathrm{CA}^{3 \mathrm{c}, 8}$, from crystallographic studies of NTD, CTD, and full-length

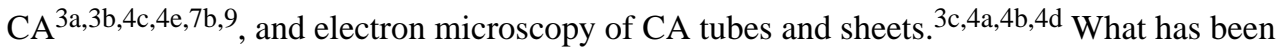
missing is high-resolution structural characterization of intermolecular interfaces within the curved, noncrystalline CA lattice that exists in mature HIV-1 capsids, for which CA tubes are an appropriate model. As demonstrated above, this missing information can be provided by solid state NMR data, including quantitative measurements of interatomic distances and bond vector orientations, conformation-dependent chemical shifts, and long-range interresidue contacts. With appropriate isotopic labeling patterns, specific interfaces can be targeted.

It is notable that the solid state NMR data lead to a unique structure for the dimerization interface, despite the variable curvature of the CA lattice and the heterogeneity of tube diameters and helicities in solid state NMR samples, and even when inequivalent cryoEM densities are used (Figs. 4D and S6). Structural plasticity in intermolecular interfaces, including the dimerization and trimerization interfaces formed by CTD domains ${ }^{3 \mathrm{c}, 4 \mathrm{~d}, 12}$, has been suggested to play a role in CA lattice curvature. Previous solid state NMR studies of CA tubes by our laboratory ${ }^{13,28}$ and by Polenova and coworkers ${ }^{12,29}$ provided evidence for dynamic disorder in the NTD-CTD linker and surrounding segments, which is consistent with variability in the intramolecular orientation between NTD and CTD. ${ }^{7 b, 30}$ The 
observation of a well-structured dimerization interface is further evidence that plasticity of intermolecular CTD-CTD interactions does not make a major contribution to the variable curvature of the mature capsid surface.

A recent crystal structure of full-length CA from Gres et al. (PDB 4XFX) ${ }^{4 \mathrm{e}}$ provides a highresolution picture of a planar lattice of $\mathrm{CA}$ hexamers, building on earlier electron diffraction studies of planar CA assemblies by Ganser-Pornillos et al. ${ }^{4 \mathrm{~b}}$ The dimerization interface in the curved lattice derived from our solid state NMR data, as described above, is very similar to the interface in the planar lattice, with differences in the precise conformations of W184 and M185 sidechains. The dimerization interface in the curved lattice is also similar to the interface in soluble CTD dimers determined by Byeon et al. (PDB 2KOD) ${ }^{3 \mathrm{c}}$ (which is also consistent with solution NMR data for full-length CA dimers ${ }^{8 e}$ ), but with somewhat larger differences in sidechain conformations and helix 9-helix 9 orientations. The dimerization interface in crystalline CTD reported by Worthylake et al. (PDB 1A43) ${ }^{3 \mathrm{~b}}$ is quite different from the interface structure in CA tubes. Dimerization interfaces in an atomic model for CA tubes developed by $\mathrm{MDFF}^{4 \mathrm{~d}}$, using the cryoEM density as a restraint, are substantially disordered (Fig. S7), in strong disagreement with our solid state NMR data.

The similarity of dimerization interface structures in noncrystalline CA tubes, determined from our solid state NMR data, and in the crystalline, planar 2D lattice of Gres et al. (PDB 4XFX) supports the idea that lattice curvature is not dependent on plasticity of the dimerization interface. Dissimilarity with the interface structure in the CTD dimer crystal structure of Worthylake et al. (PDB 1A43) may be attributable to the fact that their CTD construct included only residues 146-231, with an additional N-terminal Met residue. Similarity with the interface structure in the CTD dimer solution NMR structure of Byeong et al. (PDB 2KOD), which included residues 145-231, supports their proposal that Y145 has a significant impact on the dimerization interface.

\section{CONCLUSIONS}

We have shown that the dimerization interface, one of the key sites of intermolecular interactions within the HIV-1 CA lattice, is rigid and structurally ordered in CA tubes. From solid state NMR restraints on interatomic distances and conformations for sidechains of hydrophobic residues within the dimerization interface, supplemented by densities for helix 9 pairs from cryoEM, we have developed an atomic resolution structural model for the dimerization interface in CA tubes. Goals for future work include the acquisition of quantitative structural restraints from solid state NMR for the trimerization interface, involving intermolecular interactions of helix 10 and helix 11 segments, and for intermolecular NTD-CTD interactions that stabilize CA hexamers. Such data will lead to improved models for the complete CA lattice in noncrystalline assemblies and to a more comprehensive understanding of variable lattice curvature. The same approach can also be applied to the spherical Gag lattice of immature HIV-1, which has been proposed to involve two separate intermolecular dimerization interfaces, formed by helix 9 segments of CTD and by helix 1 segments of NTD. ${ }^{31}$ 


\section{Supplementary Material}

Refer to Web version on PubMed Central for supplementary material.

\section{Acknowledgments}

This work was supported by the Intramural Research Program of the National Institute of Diabetes and Digestive and Kidney Diseases of the National Institutes of Health, and by the NIH Intramural AIDS Targeted Antiviral Program. We thank Dr. Eric Moore for assistance with simulations of NMR data and Drs. Charles Schwieters and Guillermo Bermejo for assistance with Xplor-NIH calculations.

\section{References}

1. Ganser-Pornillos BK, Yeager M, Sundquist WI. Curr Opin Struct Biol. 2008; 18:203. [PubMed: 18406133]

2. Ganser-Pornillos BK, von Schwedler UK, Stray KM, Aiken C, Sundquist WI. J Virol. 2004; 78:2545. [PubMed: 14963157]

3. (a) Gamble TR, Yoo SH, Vajdos FF, vonSchwedler UK, Worthylake DK, Wang H, McCutcheon JP, Sundquist WI, Hill CP. Science. 1997; 278:849. [PubMed: 9346481] (b) Worthylake DK, Wang H, Yoo SH, Sundquist WI, Hill CP. Acta Crystallogr Sect D-Biol Crystallogr. 1999; 55:85. [PubMed: 10089398] (c) Byeon IJL, Meng X, Jung JW, Zhao GP, Yang RF, Ahn JW, Shi J, Concel J, Aiken C, Zhang PJ, Gronenborn AM. Cell. 2009; 139:780. [PubMed: 19914170]

4. (a) Li S, Hill CP, Sundquist WI, Finch JT. Nature. 2000; 407:409. [PubMed: 11014200] (b) GanserPornillos BK, Cheng A, Yeager M. Cell. 2007; 131:70. [PubMed: 17923088] (c) Pornillos O, Ganser-Pornillos BK, Kelly BN, Hua YZ, Whitby FG, Stout CD, Sundquist WI, Hill CP, Yeager M. Cell. 2009; 137:1282. [PubMed: 19523676] (d) Zhao GP, Perilla JR, Yufenyuy EL, Meng X, Chen B, Ning JY, Ahn J, Gronenborn AM, Schulten K, Aiken C, Zhang PJ. Nature. 2013; 497:643. [PubMed: 23719463] (e) Gres AT, Kirby KA, KewalRamani VN, Tanner JJ, Pornillos O, Sarafianos SG. Science. 2015; 349:99. [PubMed: 26044298]

5. Ehrlich LS, Liu TB, Scarlata S, Chu B, Carter CA. Biophys J. 2001; 81:586. [PubMed: 11423440]

6. Briggs JAG, Wilk T, Welker R, Krausslich HG, Fuller SD. Embo J. 2003; 22:1707. [PubMed: 12660176]

7. (a) Ganser BK, Li S, Klishko VY, Finch JT, Sundquist WI. Science. 1999; 283:80. [PubMed: 9872746] (b) Pornillos O, Ganser-Pornillos BK, Yeager M. Nature. 2011; 469:424. [PubMed: 21248851]

8. (a) Gitti RK, Lee BM, Walker J, Summers MF, Yoo S, Sundquist WI. Science. 1996; 273:231. [PubMed: 8662505] (b) Tang C, Ndassa Y, Summers MF. Nat Struct Biol. 2002; 9:537. [PubMed: 12032547] (c) Wong HC, Shin R, Krishna NR. Biochemistry. 2008; 47:2289. [PubMed: 18220423] (d) Shin R, Tzou YM, Krishna NR. Biochemistry. 2011; 50:9457. [PubMed: 21995733] (e) Deshmukh L, Schwieters CD, Grishaev A, Ghirlando R, Baber JL, Clore GM. J Am Chem Soc. 2013; 135:16133. [PubMed: 24066695]

9. (a) Momany C, Kovari LC, Prongay AJ, Keller W, Gitti RK, Lee BM, Gorbalenya AE, Tong L, McClure J, Ehrlich LS, Summers MF, Carter C, Rossmann MG. Nat Struct Biol. 1996; 3:763. [PubMed: 8784350] (b) Gamble TR, Vajdos FF, Yoo SH, Worthylake DK, Houseweart M, Sundquist WI, Hill CP. Cell. 1996; 87:1285. [PubMed: 8980234]

10. von Schwedler UK, Stray KM, Garrus JE, Sundquist WI. J Virol. 2003; 77:5439. [PubMed: 12692245]

11. Pornillos O, Ganser-Pornillos BK, Banumathi S, Hua YZ, Yeager M. J Mol Biol. 2010; 401:985. [PubMed: 20600115]

12. Byeon IJL, Hou GJ, Han Y, Suiter CL, Ahn J, Jung J, Byeon CH, Gronenborn AM, Polenova T. J Am Chem Soc. 2012; 134:6455. [PubMed: 22428579]

13. Bayro MJ, Chen B, Yau WM, Tycko R. J Mol Biol. 2014; 426:1109. [PubMed: 24370930]

14. Higman VA, Flinders J, Hiller M, Jehle S, Markovic S, Fiedler S, van Rossum BJ, Oschkinat H. J Biomol NMR. 2009; 44:245. [PubMed: 19609683] 
15. (a) Gullion T, Schaefer J. J Magn Reson. 1989; 81:196.(b) Gullion T, Baker DB, Conradi MSJ. Magn Reson. 1990; 89:479.

16. Costa PR, Gross JD, Hong M, Griffin RG. Chem Phys Lett. 1997; 280:95.

17. van Rossum BJ, de Groot CP, Ladizhansky V, Vega S, de Groot HJM. J Am Chem Soc. 2000; 122:3465.

18. Chan JCC, Tycko R. J Chem Phys. 2004; 120:8349. [PubMed: 15267757]

19. Levitt MH, Freeman R, Frenkiel T. J Magn Reson. 1982; 47:328.

20. Veshtort M, Griffin RG. J Magn Reson. 2006; 178:248. [PubMed: 16338152]

21. Bak M, Rasmussen JT, Nielsen NC. J Magn Reson. 2000; 147:296. [PubMed: 11097821]

22. Schwieters CD, Kuszewski JJ, Clore GM. Prog Nucl Magn Reson Spectrosc. 2006; 48:47.

23. Gong Z, Schwieters CD, Tang C. PLoS One. 2015; 10:e0120445. [PubMed: 25798848]

24. (a) Lorieau JL, McDermott AE. J Am Chem Soc. 2006; 128:11505. [PubMed: 16939274] (b)

Schanda P, Meier BH, Ernst M. J Am Chem Soc. 2010; 132:15957. [PubMed: 20977205]

25. De Paepe, G. Annual Review of Physical Chemistry. Johnson, MA., Martinez, TJ., editors. Vol. 63. Vol. 63. 2012. p. 661

26. (a) Sharpe S, Kessler N, Anglister JA, Yau WM, Tycko R. J Am Chem Soc. 2004; 126:4979.

[PubMed: 15080704] (b) Yang J, Weliky DP. Biochemistry. 2003; 42:11879. [PubMed: 14529300]

27. Dabbagh G, Weliky DP, Tycko R. Macromolecules. 1994; 27:6183.

28. Lu JX, Bayro MJ, Tycko R. J Biol Chem. 2016 in press.

29. Han Y, Hou GJ, Suiter CL, Ahn J, Byeon IJL, Lipton AS, Burton S, Hung I, Gor'kov PL, Gan ZH, Brey W, Rice D, Gronenborn AM, Polenova T. J Am Chem Soc. 2013; 135:17793. [PubMed: 24164646]

30. Bailey GD, Hyun JK, Mitra AK, Kingston RL. J Mol Biol. 2012; 417:212. [PubMed: 22306463]

31. Schur FKM, Hagen WJH, Rumlova M, Ruml T, Muller B, Krausslich HG, Briggs JAG. Nature. 2015; 517:505. [PubMed: 25363765] 

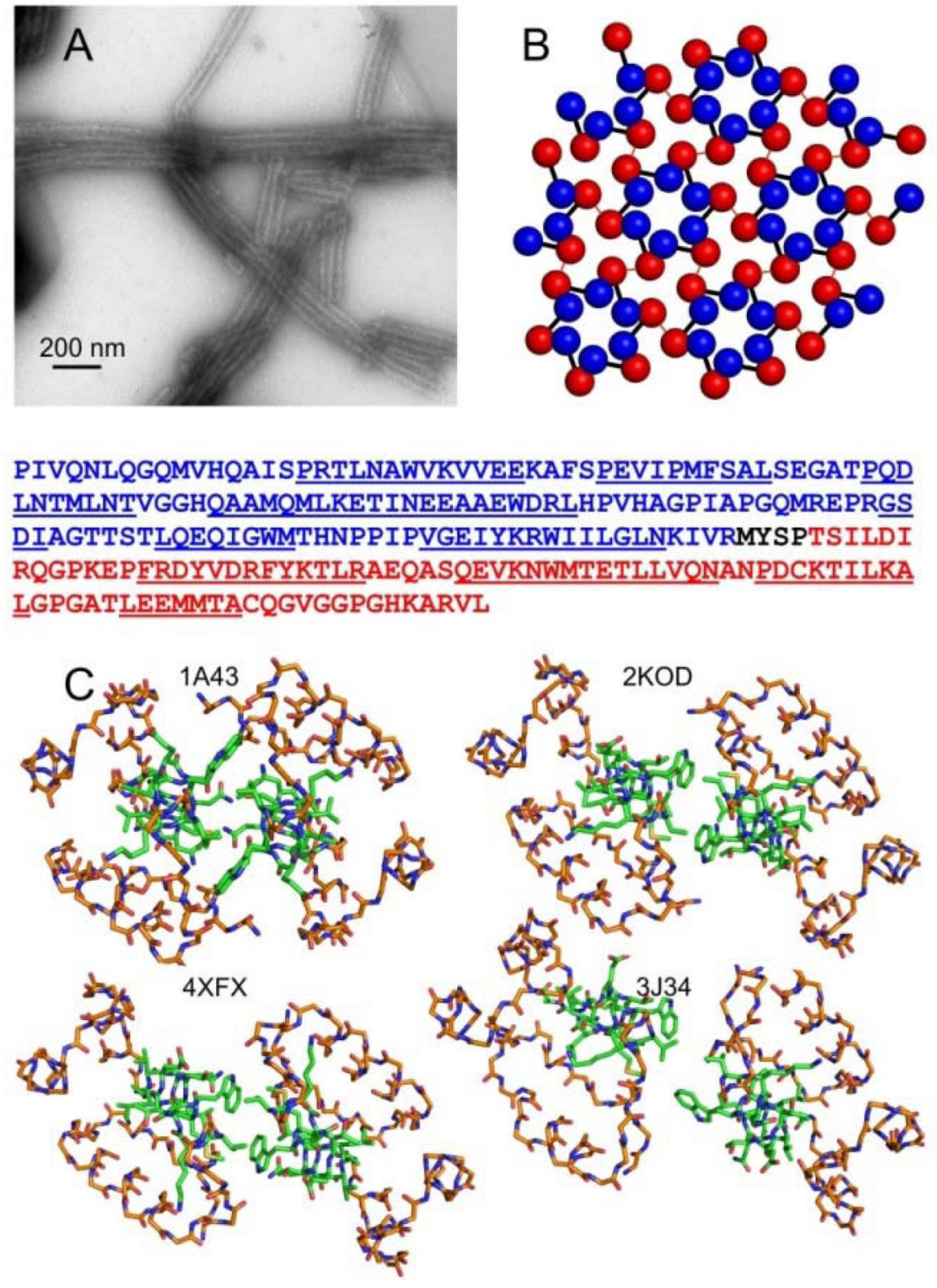

Figure 1.

Diverse dimerization interface structures from crystallography, solution NMR, and cryoEM. (A) TEM image of typical tubular HIV-1 CA assemblies studied by solid state NMR. (B) Ideal CA lattice, with NTD and CTD represented by blue and red spheres, respectively. Black bars connect NTD and CTD of the same CA molecule. Red lines represent intermolecular dimerization interfaces. The CA sequence is shown below panels $\mathrm{A}$ and $\mathrm{B}$, with the NTD in blue, the CTD in red, and helical segments underlined. (C) CTD dimer structures from the indicated PDB files, viewed down the two-fold (or approximate twofold) symmetry axes. Backbone atoms of residues 148-180 and 195-218 are shown, using orange to represent carbon atoms. Backbone and sidechain atoms of residues 181-194 are shown, using green to represent carbon atoms. For PDB 2KOD, the first of 30 NMR-based 
models is depicted. For PDB 3J34, one of the 12 cryoEM-based dimerization interfaces is depicted. 

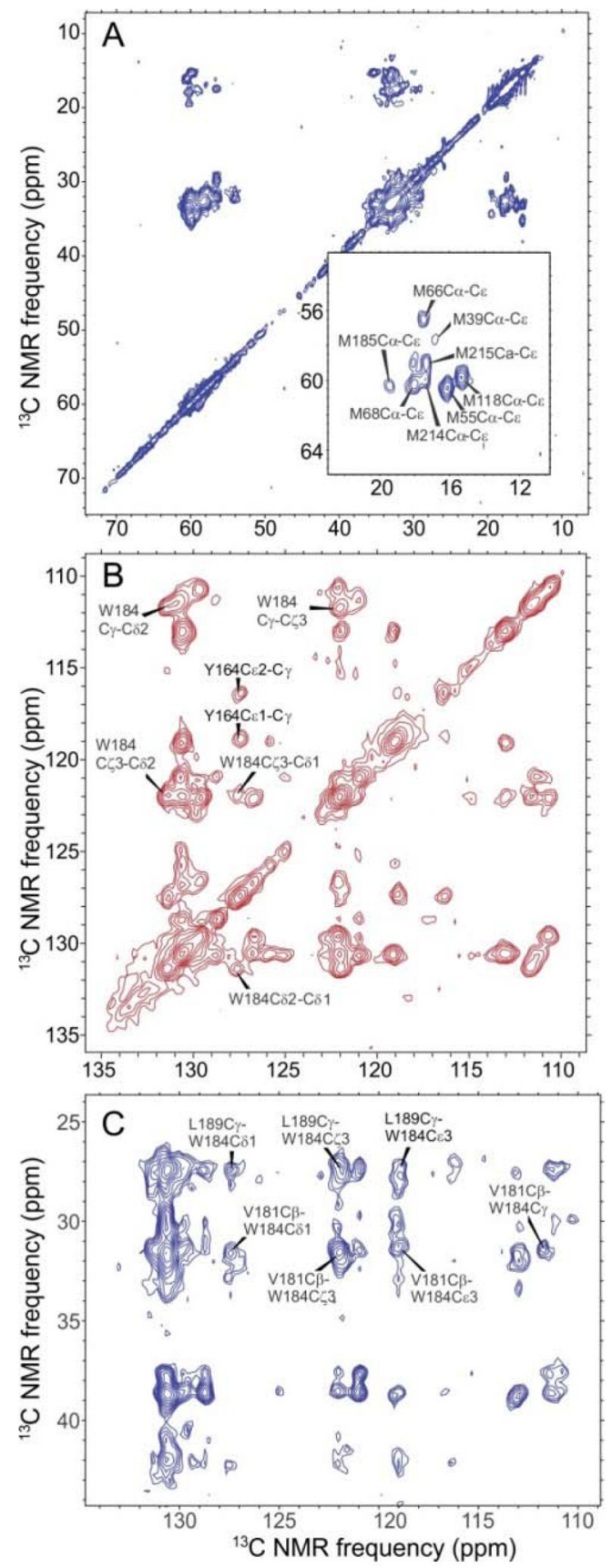

Figure 2.

2D solid state NMR spectra of HIV-1 CA tubes. (A) Aliphatic region of a $2 \mathrm{D}{ }^{13} \mathrm{C}-{ }^{13} \mathrm{C}$ spectrum of sample I, obtained with a spin diffusion mixing period $\tau_{\mathrm{SD}}=200 \mathrm{~ms}$. The inset shows assignments of Met $C_{a}-C_{\varepsilon}$ crosspeaks. (B) Aromatic region of a $2 \mathrm{D}{ }^{13} \mathrm{C}_{-}{ }^{13} \mathrm{C}$ spectrum of sample II, obtained with $\tau_{\mathrm{SD}}=700 \mathrm{~ms}$. (C) Aromatic/aliphatic region of a $2 \mathrm{D}{ }^{13} \mathrm{C}-{ }^{13} \mathrm{C}$ correlation spectrum of sample II, obtained with $\tau_{\mathrm{SD}}=700 \mathrm{~ms}$. Crosspeaks indicating long-range contacts from W184 to V181 and L189 are labeled. Intraresidue crosspeaks from the four other Trp residues (W23, W80, W117, and W133) are also 
resolved ${ }^{13}$, but for clarity are not labeled. Contour levels increase by factors of 1.2 in panels A-C. 

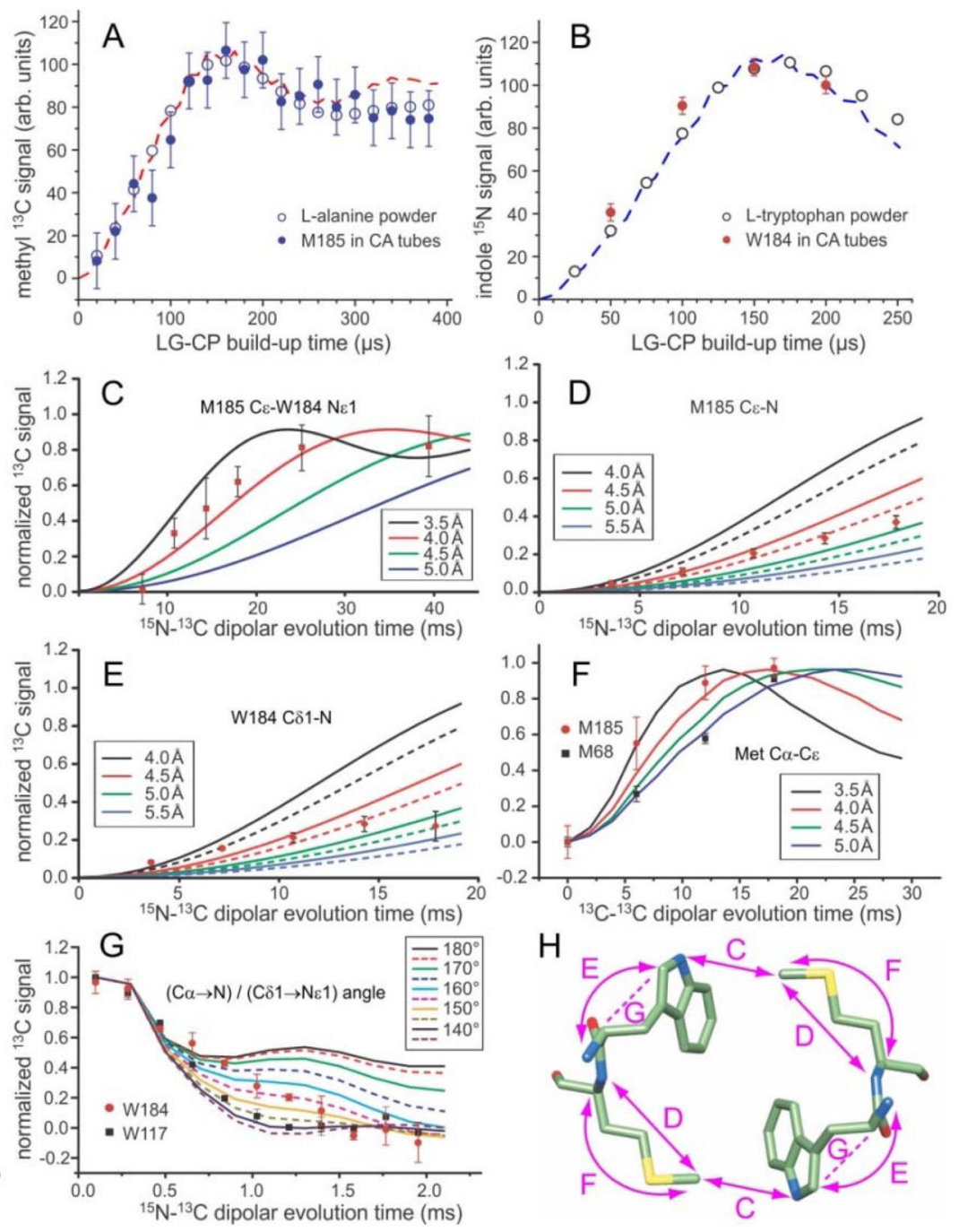

Figure 3.

Quantitative restraints on the dimerization interface structure from solid state NMR. (A,B) Comparison of ${ }^{1} \mathrm{H}_{-}{ }^{13} \mathrm{C}$ and ${ }^{1} \mathrm{H}_{-}{ }^{15} \mathrm{~N}$ LG-CP build-up data for $\mathrm{M} 185 \mathrm{C}_{\varepsilon}$ and $\mathrm{W} 184 \mathrm{~N}_{\varepsilon 1}$ in HIV-1 CA tubes with data for the methyl site in L-alanine powder and the $\mathrm{N}_{\varepsilon 1}$ site in Ltryptophan powder. The similarity of these data for CA tubes and amino acid powders indicates the absence of large-amplitude motions of M185 and W184 sidechains on submillisecond timescales. Dashed lines are ideal LG-CP simulations for four-spin (panel A) and two-spin (panel B) systems, assuming methyl C-H bond lengths of $1.08 \AA$, with rapid methyl rotation, and indole $\mathrm{N}-\mathrm{H}$ bond lengths of $0.98 \AA$. $(\mathrm{C}, \mathrm{D}, \mathrm{E}){ }^{15} \mathrm{~N}-{ }^{13} \mathrm{C}$ REDOR data that restrain the intermolecular $\mathrm{M} 185 \mathrm{C}_{\varepsilon}-\mathrm{W} 184 \mathrm{~N}_{\varepsilon 1}$ distance, the intra-residue $\mathrm{M} 185 \mathrm{C}_{\varepsilon}-\mathrm{N}$ distance, and the intra-residue $\mathrm{W} 184 \mathrm{C}_{\varepsilon 1}-\mathrm{N}$ distance in HIV-1 CA tubes. Solid curves in panel $\mathrm{C}$ are ideal two-spin simulations for isolated ${ }^{15} \mathrm{~N}-{ }^{13} \mathrm{C}$ pairs with the indicated distances. Solid and dashed curves in panels $\mathrm{D}$ and $\mathrm{E}$ are three-spin simulations, including an additional ${ }^{15} \mathrm{~N}$ at the farthest and closest possible distance (see text). (F) BroBaRR data that restrain the intra-residue $\mathrm{C}_{\mathrm{a}}-\mathrm{C}_{\varepsilon}$ distance in M185. Data for M68 are also shown for comparison. Solid lines are two-spin simulations for the indicated ${ }^{13} \mathrm{C}-{ }^{13} \mathrm{C}$ distances. (G) 
NCCN data that restrain the angle between the $\mathrm{C}_{\mathrm{a}}-\mathrm{N}$ and $\mathrm{C}_{\delta 1}-\mathrm{N}_{\varepsilon 1}$ bond vectors in W184. Data for W184 are also shown for comparison. Solid and dashed lines are ideal simulations for the indicated angles. (H) Dimer of W184-M185 units, showing the distances (doubleheaded arrows) and vector angles (dashed lines) restrained by data in panels C-G. Error bars in panels $\mathrm{A}-\mathrm{G}$ represent uncertainties calculated from the root-mean-squared noise in the corresponding solid state NMR spectra. 

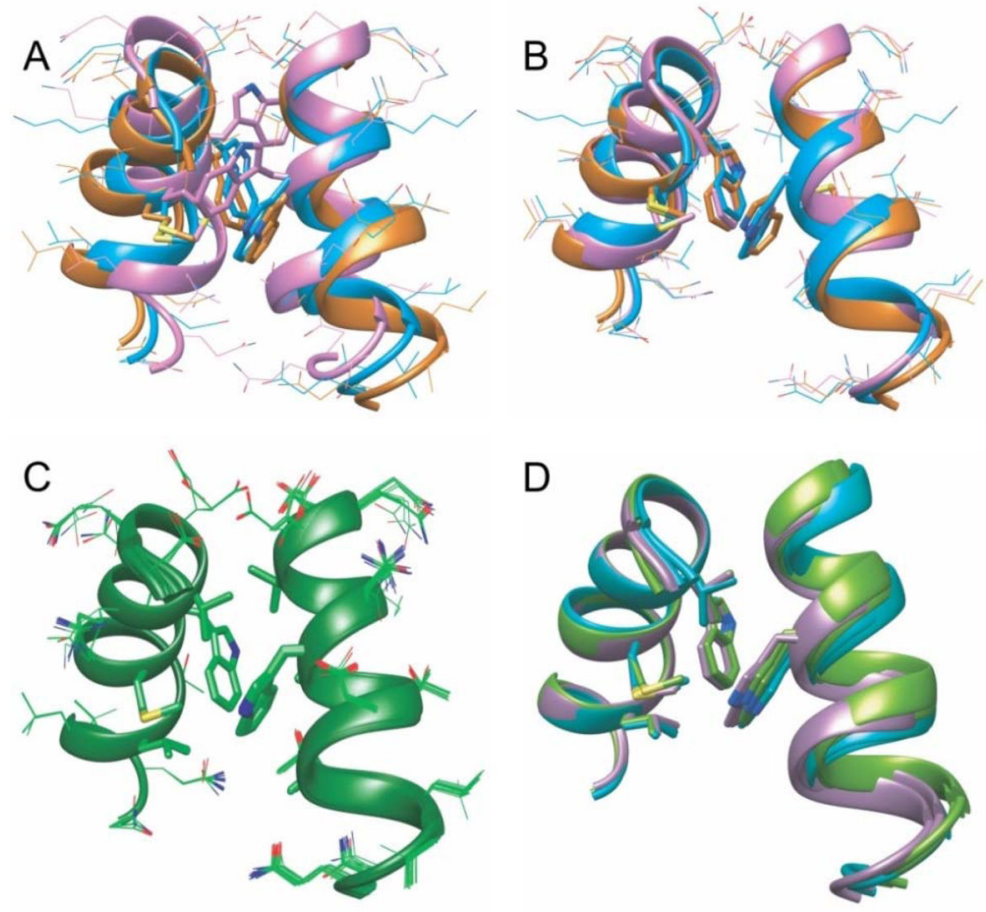

Figure 4.

Structure refinement based on solid state NMR restraints and cryoEM densities. (A) Superposition of residues 178-193 in three different starting structures, taken from studies of crystalline full-length CA (PDB 4XFX, orange), CTD dimers in solution (PDB 2KOD, cyan), and crystalline CTD dimers (PDB 1A43, pink). Alignment of the three structures highlights differences in the relative helix orientations and the positions of W184 and M185 sidechains. Other amino acid sidechains are represented by thinner sticks. (B) Final structures obtained from the three starting structures in panel A, with the same color scheme. Lowest-energy structures are shown from three sets of simulated annealing calculations, using the structural restraints from solid state NMR data in Figs. 2 and 3 and cryoEM densities from one of the dimer interfaces in EMD-5582. (C) Superposition of 20 lowenergy structures from 100 independent calculations, using the dimer interface from PDB 4XFX as the starting structure. Sidechains of W184, M185, V181, and L189 are represented by thick sticks. (D) Superposition of six lowest-energy final structures from calculations that used cryoEM densities from six different dimer interfaces in EMD-5582. Starting structures were taken from PDB 4XFX. Based on two-fold symmetry in the cryoEM reconstruction, the dimer interface densities are grouped into three pairs, and the resulting refined structures are colored purple, cyan, and green. The six final structures are optimally aligned for one helix, on which sidechains of W184, M185, V181, and L189 are shown. Only W184 is shown on the other helix. 


\section{Table 1}

HIV-1 CA samples for solid state NMR

\begin{tabular}{|c|c|c|}
\hline HIV-1 CA sample & labeling pattern & experimental purpose \\
\hline I & $\mathrm{U}-{ }^{15} \mathrm{~N},{ }^{13} \mathrm{C}-\mathrm{Met}$ and $\mathrm{U}-{ }^{15} \mathrm{~N}$ & Met chemical shift assignments, Met BroBaRR \\
\hline II & $2-{ }^{13} \mathrm{C}$-glycerol and $\mathrm{U}-{ }^{15} \mathrm{~N}$ & $\begin{array}{l}\text { Trp chemical shift assignments, long-range aromatic/aliphatic } \\
\text { correlations }\end{array}$ \\
\hline III & $\begin{array}{l}2-{ }^{13} \mathrm{C} \text {-glycerol, } \mathrm{U}_{-}{ }^{15} \mathrm{~N} \text {, unlabeled Tyr, and unlabeled } \\
\text { Phe }\end{array}$ & $\begin{array}{l}\text { Trp chemical shift assignments, long-range aromatic/aliphatic } \\
\text { correlations, Trp NCCN }\end{array}$ \\
\hline IV & ${ }^{13} \mathrm{C}_{\varepsilon}$-Met or ${ }^{15} \mathrm{~N}$-indole (1:1 mixture) & LG-CP, intermolecular REDOR \\
\hline $\mathrm{V}$ & ${ }^{13} \mathrm{C}_{\varepsilon}-\mathrm{Met}^{a}, 2-{ }^{13} \mathrm{C}$-indole, and $\mathrm{U}-1{ }^{15} \mathrm{~N}$ & intramolecular REDOR \\
\hline
\end{tabular}

${ }^{a}$ Although the precursor methionine was not ${ }^{15} \mathrm{~N}$-labeled, aminotransferase-catalyzed amino exchange results in incorporation of $15_{\mathrm{N}}$ at amide sites of Met residues. 